\title{
Distinct Recovery Length and Multiple 1-RM Attempts for Both Upper and Lower Body Exercises
}

\author{
Estevão Scudese ${ }^{1,2,3}$, Adolf Grotz', Cristiano Queiroz de Oliveira', Fabiana Rodrigues \\ Scartoni $^{2,3}$, Alan Bruno Silva Vasconcelos ${ }^{4}$, Marzo Edir Da Silva-Grigoletto ${ }^{4,5}$, Estélio \\ Henrique Martin Dantas ${ }^{3}$ and Gilmar Weber Senna ${ }^{1,2,3^{*}}$
}

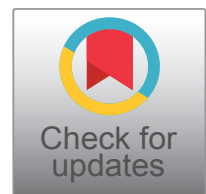

${ }^{1}$ Health Science Center, Petrópolis Catholic University, Brazil

${ }^{2}$ Sports and Exercise Science Laboratory (LaCEE), Petrópolis Catholic University, Brazil

${ }^{3}$ Biosciences and Human Movement Laboratory (LABIMH), Federal University of State of Rio de Janeiro (UNIRIO), Brazil

${ }^{4}$ Post-graduation Program in Physiological Sciences, Federal University of Sergipe, Brazil

${ }^{5}$ Post-graduation Program in Physical Education, Federal University of Sergipe, Brazil

*Corresponding author: Dr. Gilmar Weber Senna, Federal University of State of Rio de Janeiro (UNIRIO), Rua 16 de março, 167, Apto 801, Centro, Petrópolis/RJ, 25620040 - Petrópolis, RJ, Brazil

\begin{abstract}
The present study aims to verify the responses of different rest period lengths on multiple attempts of the 1-RM (repetition maximal) in the Bench Press (BP) and Leg-Press (LP) exercises. Fifteen trained men $(83.38 \pm 10.89 \mathrm{~kg}, 179.40 \pm$ $6.70 \mathrm{~cm}, 22.40 \pm 2.52$ years, $11.93 \pm 3.51 \% \mathrm{BF}, 1.23 \pm 0.16$ $\mathrm{kg}$ relative strength [maximum bench press load] $\mathrm{kg}$ [body weight ${ }^{-1}$ ) performed a test and retest for load evaluation in both exercises. After load determination, subjects performed four more visits, with five 1-RM attempts with distinct intervals (30 seconds, 1, 2, and 5 minutes). The Cochran $Q$ test was applied in order to verify significant differences between 1-RM attempts. The results shown that short rest periods (<2-min) promoted a decrease in the performance of multiple attempts, and that longer rests (2 to 5-min) were the best option for maintaining performance in 1-RM sets for both exercises. More specifically, 2 minutes was ideal for the maintenance of up to three successfully attempts and 5 minutes proved to be ideal for four attempts and somewhat high around $70 \%$ of success for the fifth and final set. We can conclude that the 2- and 5-minute rest intervals are the best option for maintaining multiple 1-RM attempts, and the choice must depend on the pre-stipulated number of sets.
\end{abstract}

\section{Keywords}

Weightlifting, Muscle strength, Exercise

\section{Introduction}

The maximum repetition test (1-RM) has been used to verify the maximum force since the early days of resistance exercise scientific investigations $[1,2]$ and is widely used as the gold standard measure for strength according to the American College of Sports Medicine (ACSM) [3].

When the goal is to increase levels of muscle strength and power, the ACSM suggests that 1-RM loads should be implemented in regular athlete training routine [4]. Additionally, when designing a resistance exercise program, one should consider the manipulation of several methodological variables that might influence training outcomes, both acutely and chronically [4]. One of these variables that are crucial to strength training is the rest interval between sets already known to influence the metabolic, hormonal, cardiovascular, and neuromuscular responses [5-11].

Although the current ACSM recommendation [3] is to apply longer recovery lengths for 1-RM testing attempts (such as three to five minutes), the current scientific literature reports that it is possible to reproduce a single subsequent attempt with a shorter recovery, which could maintain performance but increase train-

Citation: Scudese E, Grotz A, de Oliveira CQ, Scartoni FR, Vasconcelos ABS, et al. (2020) Distinct Recovery Length and Multiple 1-RM Attempts for Both Upper and Lower Body Exercises. Int J Sports Exerc Med 6:176. doi.org/10.23937/2469-5718/1510176

Accepted: September 30, 2020; Published: October 02, 2020

Copyright: (C) 2020 Scudese E, et al. This is an open-access article distributed under the terms of the Creative Commons Attribution License, which permits unrestricted use, distribution, and reproduction in any medium, provided the original author and source are credited. 
ing efficiency or test for loads of maximum intensity $[5,12,13]$.

For instance, in 1994, Weir, et al. [13] conducted the first study that investigated different rest lengths implications on the maximum horizontal Bench Press (BP) performance and concluded that the shorter rest (1-minute) was already sufficient for a successful second bench press maximal attempt. In another study comparing the effect of different intervals for two 1-RM attempts on the barbell squat exercise, Matuszak, et al. [12] found that for the shorter 1-minute rest interval, 13 of 17 participants successfully performed the second attempt (75.4\%); for the interval of 3 minutes, 16 of 17 (94.1\%) and the interval of 5 minutes, 15 of 17 (88.2\%) completed the second squat attempt.

In a recent study, Scudese, et al. [5] compared short recovery lengths (20, 40 and 60 seconds) for two subsequent 1-RM attempts in the BP exercise. The authors found showed that most individuals were able to perform the 1-RM in a subsequent attempt of 20,40, and 60 seconds apart. These results, in part, endorse the data found by Weir, et al. [13] indicating that as in BP, very short rest may be sufficient for recovery between two subsequent attempts in the 1-RM.

Although the indicated studies show similar results, the previous investigations presented only two 1-RM attempts to verify the optimal interval for performance maintenance. In this regard, very little is known about multiple 1-RM attempts, designed like a training routine, as previously suggested as a strategy for increasing maximal strength [4]. In order to attend this issue, the main goal of this current investigation is to verify an optimal recovery length for multiple (five) consecutive 1-RM attempts.

\section{Methods}

\section{Subjects}

Fifteen trained men $(83.38 \pm 10.89 \mathrm{~kg} ; 179.40 \pm$ $6.70 \mathrm{~cm} ; 22.40 \pm 2.52$ years; $11.93 \pm 3.51 \%$ body fat; $1.23 \pm 0.16 \mathrm{~kg}$ relative strength [bench press maximum load] $/ \mathrm{kg}_{\text {[body weight] }}{ }^{-1}$ ) with at least one year of resistance training experience. After the initial anamnesis, the following inclusion criteria were adopted in order to standardize the subjects' selection: a) Minimum of four times a week of training frequency, lasting approximately one hour each session, and rest intervals between the series which varied from one to two minutes; b) No use of any ergogenic resource that would improve performance; c) Absence of any acute or chronic injuries that might affect performance; and d) To avoid any intense physical activity during the experimental procedures. After the selection based on the inclusion criteria, all participants answered negatively to all PAR-Q questions [14]. Besides, all subjects read and signed a consent form, following the Declaration of Helsinki, and all study procedures were previously approved by the ethics committee of the Petrópolis Catholic University, number: 69419317.3.0000.5281. The study followed all the norms of the Brazilian National Health Council (CNS), according to the 510/2016 resolution.

\section{One repetition maximum determination (1-rm)}

After two weeks of familiarization, the 1-RM test and retest were performed on two non-consecutive days in the BP exercise and then on another two non-consecutive days in the Leg Press (LP) exercise. If individuals were unable to complete a repetition with the previously stipulated load, adjustments would be made, and a retry would be performed with the interval of three to five minutes with a maximum of five attempts per visit. To minimize measurement errors, the following strategies were adopted: (a) Standardized instructions for experimental procedures were provided to the participants prior to the experiments; (b) Individuals received standardized instructions in the exercise technique; (c) The position of the body was kept constant; (d) A verbal stimulus was provided during the exercises in order to compensate for the maximum effort of each subject [14], and the mass of all plates and bars used were determined using a precision scale [6]. The greatest load lifted during the two test sessions for each exercise was recorded as the 1-RM load. Before each load test for both exercises, a warm-up was conducted consisting of a single set of twelve repetitions at $40 \%$ of 1-RM [8].

\section{Experimental procedures}

Forty-eight hours after the load tests, the experimental protocol was divided into four visits for each exercise, and participants performed a distinct rest period on each non-consecutive day. The order of the exercises and the rest protocol between the trials (30 seconds, 1,3 , or 5 minutes) was selected for each subject using the Latin square technique. Before the start of each day of the experiment, a warm-up was performed, with a single set with $40 \%$ of the load of 1-RM for twelve repetitions in BP and LP. At the first visit, all subjects were reported on the interval between attempts they were to perform. At the second visit, subjects performed the same procedures with another selected rest condition. On the third and fourth visit, the previous procedures were followed with the stipulated rest protocol. The test would be discontinued if any of the following conditions occurred: If the participant reached the concentric failure; was not able to produce force in order to move the resistance in the concentric phase; and if the bar remained on isometric contraction for more than two seconds. The subjects were instructed to perform the concentric and eccentric phases of the movement without interruptions, with the repetition velocity being self-selected, alongside with two experienced instructors supervised all the experimental sessions.

\section{Statistical analysis}

In order to evaluate possible differences between 
the 1-RM test and retest, an intra-class correlation test was performed for both exercises (BP and LP). The Cochran $Q$ test was applied in order to verify significant differences between 1-RM trials with binary characteristics (0 or 1 ) that signify failures or successes in each attempt. Besides, multiple comparisons in pairs were used to analyze different differences between multiple attempts (five trials). SPSS software version 21.0 was used for statistical analysis (IBM, Inc). The significance adopted was $\mathrm{p} \leq 0.05$.

\section{Results}

Excellent correlations between test $(102.00 \pm 11.37$ $\mathrm{kg})$ and retest $(103.69 \pm 11.14 \mathrm{~kg})$ of $1-\mathrm{RM}$ were verified $(r=0.99)$ for BP and test $(350.06 \pm 42.62 \mathrm{~kg})$ and retest $(357.13 \pm 42.62 \mathrm{~kg})$ of $1-\mathrm{RM}$ were verified $(r=0.98)$ for LP.

The results for the $\mathrm{BP}$ indicated that the 30-second interval revealed a significant reduction for the third, fourth, and fifth attempts ( $p<0.0001$ ) compared to the first set. Similar reductions occurred for the 1-minute interval ( $p<0.0001)$ compared to the initial attempt. Also for the 1-minute length, reductions occurred concerning the second attempt for the third $(p=0.011)$, fourth and fifth attempts $(p=0.003)$. For the 2 -minute interval, the significant decrease occurred on the fifth attempt com- pared to the first, second, and third trials ( $p<0.0001$ ). In the 5-minute interval, significant differences were observed for the first $(p=0.019)$ and second $(p=0.019)$ for the fifth 1-RM set.

In the comparison of different intervals between sets, the $2(p=0.01)$ and $5(p=0.0001)$ minutes recoveries were more efficient when compared to the shorter thirty seconds, as early as for the second attempt. In the third set, the intervals of 30 seconds and one minute presented a reduced performance when compared to 2 $(p<0.0001)$ and 5 minutes (thirty seconds, $p<0.0001$; one $\min , p=0.001)$. On the fourth set, the 5 -minute interval showed better performance compared to 30 -second intervals $(p<0.0001)$ and 1 minute $(p=p<0.0001)$. Finally, for the fifth trial, 5 minutes of intervals showed significant differences over all other intervals (30 seconds, $p<0.0001$; 1-min, $p<0.0001 ; 2-\min , p=0.005)$. All data is represented in detail in Figure 1.

The results obtained for the LP showed that the 30-second interval obtained a significant reduction for the second $(p=0.005)$ third, fourth and fifth sets $(p<$ $0.0001)$ compared to the initial attempt. Significant reductions occurred for the 1-minute interval between the fourth $(p<0.0001)$ and fifth trials $(p<0.0001)$ compared to the initial and fourth sets $(p=0.001)$ and fifth

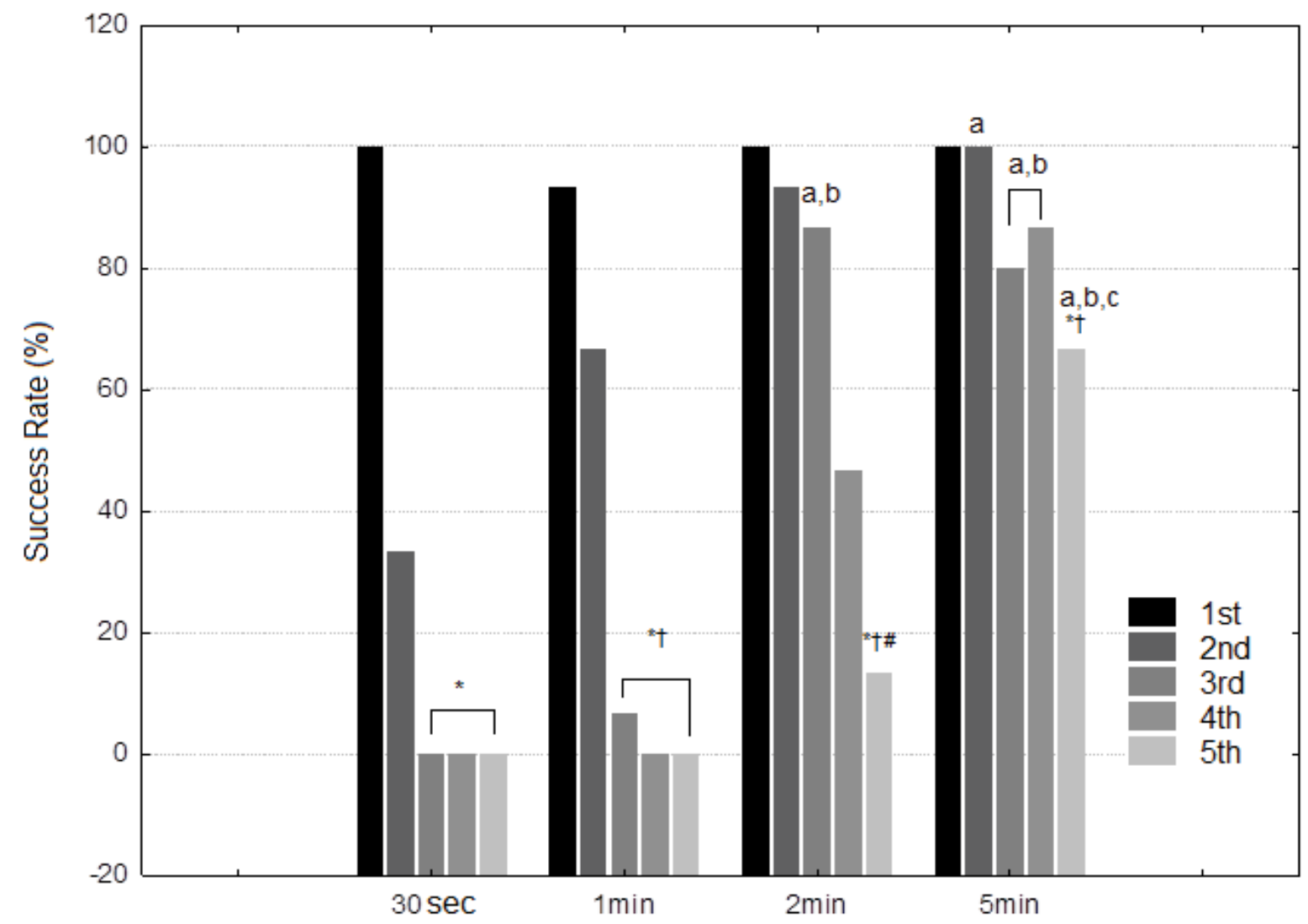

Figure 1: Success rate for multiple 1-RM attempts at different interval conditions in the horizontal barbell bench press exercise.

"Significant difference compared to the first set; †Significant difference compared to the first set; \#Significant difference compared to the first set; aSignificant difference compared to the $30 \mathrm{sec}$ rest length; 'Significant difference compared to the 30 sec rest length; 'Significant difference compared to the $30 \mathrm{sec}$ rest length. 


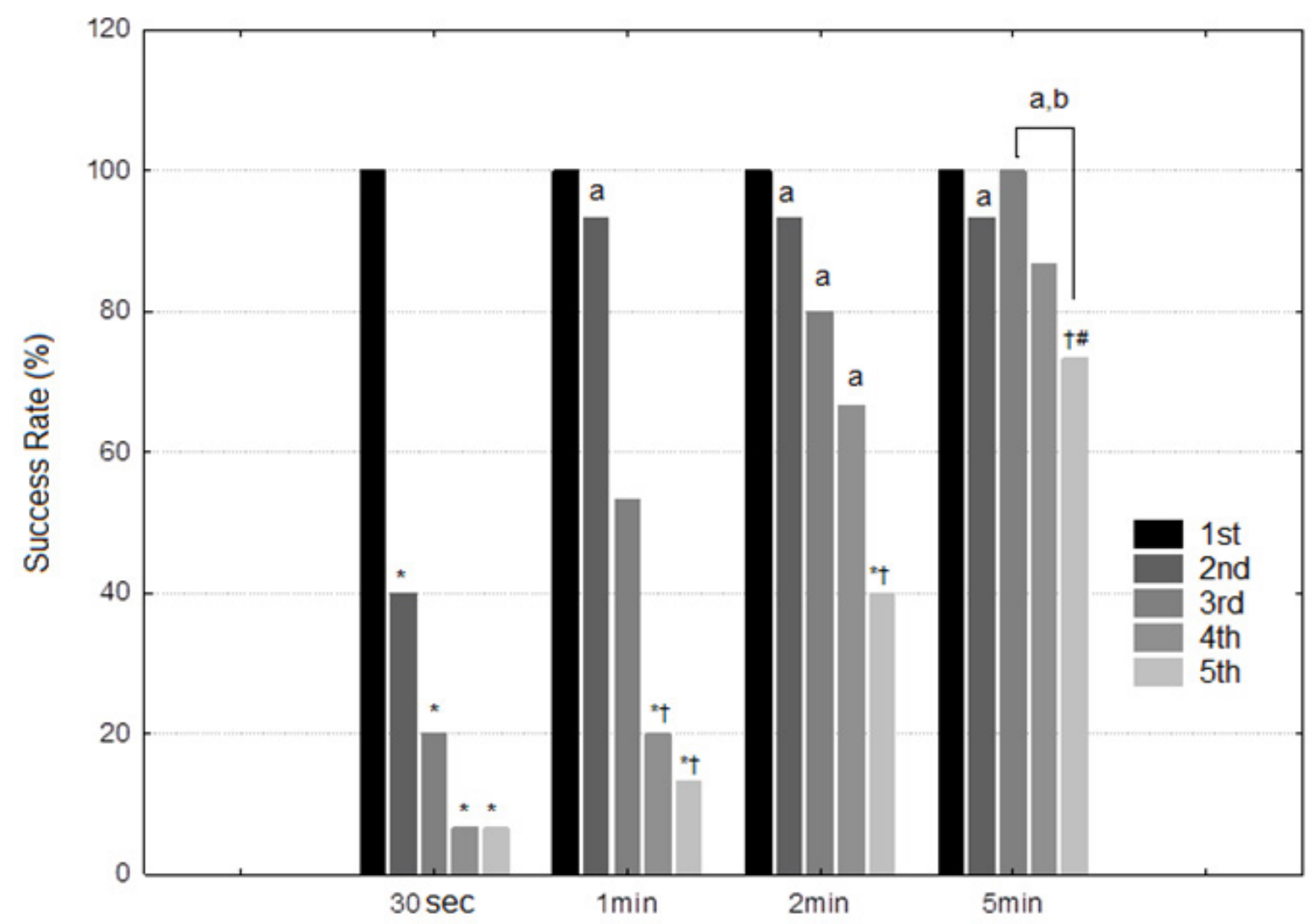

Figure 2: Success rate for multiple 1-RM attempts at different interval conditions in the barbell squat exercise.

"Significant difference compared to the first set; ${ }^{+}$Significant difference compared to the first set; "Significant difference compared to the first set; a'Significant difference compared to the $30 \mathrm{sec}$ rest length; 'bignificant difference compared to the 30 sec rest length.

set $(p<0.0001)$ compared to the second set. For the 2-minute interval, the significant decrease occurred on the fifth attempt compared to the first $(p<0.0001)$ and second ( $p=0.003)$. In the 5-minute interval, significant differences were observed for the first $(p=0.047)$ and third ( $p=0.047$ ) relative to the fifth 1-RM set.

In the comparison of different intervals between the sets, the intervals of one $(p=0.001), 2(p=0.001)$ and 5 $(p=0.001)$ minutes were more efficient when compared to 30 seconds on the second attempt. On the third set, the 30 -second rest $(p<0.0001)$ and 1 minute $(p=0.005)$ showed a reduced efficiency compared to 5 minutes rest. On the fourth set, the 5-minute interval was shown to perform best compared to 30-second intervals ( $p<$ $0.0001), 1$ minute ( $p=0.004)$ and 30 seconds and 2 minutes $(p=0.012)$. For the fifth set, 5 minutes of intervals showed significant differences over shorter intervals (thirty sec, [ $=0.001]$; one $\min ,[p=0.003]$ ) Figure 2.

\section{Discussion}

Our findings made evident that short intervals, such as thirty seconds and one minute were not enough to maintain performance for multiple 1-RM attempts for both upper and lower limbs. In contrast, the results for the longer intervals ( 2 and 5 minutes), seemed sufficient for the successful maintenance of multiple attempts performance. More specifically, one should note that the prescription of the rest length for 1-RM sets must be designed with the total volume of successful intended sets in mind. For instance, we found that two minutes might be sufficient to achieve a total of three sets, but if the goal is to conclude more sets, we verified that the longer 5-minute rest range was optimal for maintaining four and even five sets (around 70\% of success for the fifth set).

Although current scientific literature regarding the possible rest interval length for 1-RM attempts proves the possibility of reproducing a single subsequent try with a very short rest (around 1 minute) $[6,12]$, the ACSM [3] recommends that long intervals (3 to 5 minutes) must be implemented for performance maintenance for maximal strength purposes. The results obtained in this experiment are in accordance with the ACSM recommendation [3]. In this experiment, the hypothesis that long intervals should be implemented when designing a strength-oriented goal in mind, and even more relevant if the intended volume is higher. However, if a number of suggested attempts are less than or equal to three sets, the 2-minute interval appears to be sufficient.

These data seem to be in line with the few reports in the literature on the subject $[5,12,13]$. More specifically, Weir, et al. [13] examined the effect of different intervals on performance during repeated attempts of the 1-RM test in BP. Sixteen trained men participated in the study. At the first visit, the load for 1-RM was determined. The following four experiment sessions involved 
the execution of two trials with 1-RM loads, with different recovery times $(1,3,5$, and 10 minutes). All intervals were sufficient when the second attempt was made, allowing approximately the same number of participants to complete the second attempt.

Additionally, in an experiment with the very similar method but this time for lower limb exercise, Matuszak, et al. [12] observed the effects of different recovery intervals on repeated 1-RM attempts in barbell squat exercise. Seventeen trained men participated in the study. The load for 1-RM was established through test and retest performed on different days. Subsequently, three sessions were performed involving two 1-RM attempts, with intervals between 1,3 , or 5 minutes. The results demonstrated that for 1-minute rest interval, 13 of 17 participants successfully performed the second attempt (75.4\%); for 3 minutes, 16 of 17 (94.1\%) and for 5 minutes, 15 of 17 completed (88.2\%). The results presented by Matuszak, et al. [12] corroborate with Weir, et al. [13] findings, indicating that independently of the exercised body part, one-minute of rest might be sufficient to recover for a single subsequent 1-RM trial for multijoint exercises. Both studies make similar comparisons relating rest and 1-RM attempts at multi-joint exercises.

In a recent study, Scudese, et al. [5] investigated the effect of short intervals between two BP exercise attempts. Eighteen trained men participated in the study, where they have performed three sessions with different rest lengths of 20, 40 and 60 seconds, between two 1-RM consecutive BP attempts. Surprisingly the authors found that in rest intervals of 20 and 60 seconds, $88.88 \%$ of the subjects were able to perform the second attempt, and for the rest interval of 40 seconds, $83.33 \%$ successfully repeated the 1-RM, without a significant difference between the obtained data. Thus, it can be stated that it is possible to reproduce a 1-RM bout with very short rest lengths between the attempts. Even with these experiments corroborating their findings, there is still a gap that remains related to the effect of short and long rests for three or more 1-RM attempts.

Other experiments have proposed to investigate the differences of different intervals with intense near-maximal loads $[6,8]$. More specifically, In a previous pioneer investigation conducted by Scudese, et al. [6] the authors compared different intervals $(1,2,3$ and 5 minutes) for five sets of 3-RM in BP exercise. This study was the first experiment that focused on distinct rest periods for this load range (3-RM). The authors found that there was a significant difference when compared to the intervals of 2,3 , and 5 minutes compared to the shorter 1 minute. When performing the shorter rest period (1-minute), there was a significant decrease in performance already being observed from early at the second attempt. It can be concluded that short intervals (1 minute) show a significant decrease in performance when compared to long intervals (2, 3 and 5 minutes) for a near-maximal lad at 3-RM.

In a more recent investigation, Senna, et al. [8] compared different rest periods (1, 2, 3 and 5 minutes) for five sets of 3-RM in the BP exercise and chest fly machine. The authors found that the rest for multi- and single-joint exercises should be designed differently when 3-RM loads are prescribed. The 2-minute rest seemed ideal for performance maintenance in single-joint exercises, and the 3-minute interval was better for multijoint exercises. These studies corroborate partially with the experiments performed, showing that short intervals promote a significant performance drop when compared to long intervals, either for 1 or 3-RM. Another factor to be considered is that the prescription of the interval for multiple 1-RM attempts depends directly on the number of series to be performed by the individual and the exercise type (multi or single-joint).

\section{Conclusion}

Based on our findings, we concluded that the ideal rest interval length for maintaining performance for multiple 1-RM sets would depend on the number of pre-designed attempts. For instance, the longer five-minute rest interval allowed subjects to successfully perform four sets with maximum loads for both upper and lower body, and even a fifth attempt with a somewhat high success rate (around 70\%). However, if the goal is to perform fewer sets in a time-efficient manner, the two-minute interval appears to be sufficient for performing at least three successful 1-RM attempts for both upper and lower body. It is essential to highlight that this data was obtained from recreationally trained individuals (non-athletes), and more studies are needed to expand our knowledge on the effect of different intervals for multiple 1-RM attempts in different models such as single-joint exercises and different populations.

\section{Financial Support}

This study was financed in part by the Coordenação de Aperfeiçoamento de Pessoal de Nível Superior - Brasil (CAPES) - Finance Code 001.

\section{Authors Contributions}

ES, EHMD and GWS designed research; AG, CQO and ABSV performed experiments; ES and FRS analyzed data; ES, EHMD, MESG and GWS interpreted results of experiments; ES, AG and CQO drafted manuscript; ABSV and FRS edited and revised manuscript; ES, EHMD, MESG and GWS approved final version of manuscript.

\section{References}

1. Berger $R$ (1961) Determination of the resistance load for 1-RM and 10-RM. J Assoc Physi Ment Rehab 15: 108-110.

2. RA Berger (1962) Optimum repetitions for the development of strength. Res Q 33: 334-338.

3. American College of Sports Medicine, Riebe D, Ehrman JK (2018) ACSM's guidelines for exercise testing and prescrip- 
tion, Tenth edition. Wolters Kluwer, Philadelphia.

4. American College of Sports Medicine (2009) Progression Models in Resistance Training for Healthy Adults: Med Sci Sports Exerc 41: 687-708.

5. Scudese E, Senna G, Queiroz C, Dantas EHM, Simao R, et al. (2016) Influence of very short rest period lengths on repeated one maximun repetition bench press performance. Rev Andal Med Deporte S1888754616300594.

6. Scudese E, Willardson JM, Simão R, Senna G, de Salles BF, et al. (2015) The Effect of Rest Interval Length on Repetition Consistency and Perceived Exertion During Near Maximal Loaded Bench Press Sets. J Strength Cond Res 29: 3079-3083.

7. Senna G, Queiroz de Oliveira C, Kreuger S, Scudese E, Monteiro W, et al. (2016) Hypotensive Effect of Resistance Training Performed on Stable vs. Unstable Surfaces. J Exerc Physiol 19: 17-27.

8. Senna GW, Willardson JM, Scudese E, Simao R, Queiroz C, et al. (2016) Effect of Different Interset Rest Intervals on Performance of Single and Multijoint Exercises with Near-Maximal Loads. J Strength Cond Res 30: 710-716.
9. Senna G, Figueiredo T, Scudese E, Baffi M, Carneiro F, et al. (2012) Influence of Different Rest Interval Length in Multi-Joint and Single-Joint Exercises on Repetition Performance, Perceived Exertion, and Blood Lactate.

10. Senna G, Scudese E, Carneiro F, Torres JB, Queiroz C, et al. (2015) Multi-Joint and Single-Joint Exercise Performance and Perceived Exertion with Several Different Recoveries. J Exerc Physiol 18: 91-100.

11. Senna G, Scudese E, Luiz Martins C, Scartoni FR, Carneiro F, et al. (2016) Rest Period Length Manipulation on Repetition Consistency for Distinct Single-Joint Exercises. J Exerc Physiol 19: 93-101.

12. Matuszak ME, Fry AC, Weiss LW, et al. (2003) Effect of rest interval length on repeated 1 repetition maximum back squats. J Strength Cond Res 17: 634-637.

13. Weir J, Wagner L, Housh T (1994) The Effect of Rest Interval Length on Repeated Maximal Bench Presses. J Strength Cond Res 8: 58-60.

14. Haff G, Triplett NT, National Strength \& Conditioning Association (U.S.) (2016) Essentials of strength training and conditioning. Fourth edition. Human Kinetics, Champaign, IL. 